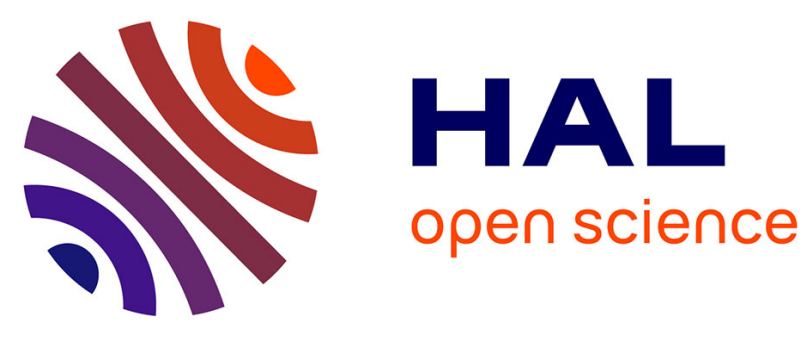

\title{
A forest canopy as a living archipelago: Why phylogenetic isolation may increase and age decrease diversity
}

Jose Hidasi-Neto, Richard Bailey, Chloe Vasseur, Steffen Woas, Werner Ulrich, Olivier Jambon, Ana M. C. Santos, Marcus Cianciaruso, Andreas Prinzing

\section{To cite this version:}

Jose Hidasi-Neto, Richard Bailey, Chloe Vasseur, Steffen Woas, Werner Ulrich, et al.. A forest canopy as a living archipelago: Why phylogenetic isolation may increase and age decrease diversity. Journal of Biogeography, 2019, 46 (1), pp.158-169. 10.1111/jbi.13469 . hal-02049181

HAL Id: hal-02049181

https://hal-univ-rennes1.archives-ouvertes.fr/hal-02049181

Submitted on 2 Sep 2020

HAL is a multi-disciplinary open access archive for the deposit and dissemination of scientific research documents, whether they are published or not. The documents may come from teaching and research institutions in France or abroad, or from public or private research centers.
L'archive ouverte pluridisciplinaire HAL, est destinée au dépôt et à la diffusion de documents scientifiques de niveau recherche, publiés ou non, émanant des établissements d'enseignement et de recherche français ou étrangers, des laboratoires publics ou privés. 


\section{2 why phylogenetic isolation may increase and age decrease diversity}

4 Short title: Canopies as living archipelagos

5

6

Jose Hidasi-Neto ${ }^{1}$, Richard I. Bailey ${ }^{2}$, Chloe Vasseur ${ }^{3}$, Steffen Woas ${ }^{4}$, Werner Ulrich $^{6}$,

Olivier Jambon ${ }^{6}$, Ana M.C. Santos ${ }^{7}$, Marcus V. Cianciaruso ${ }^{1}$, Andreas Prinzing $^{6 *}$

${ }^{1}$ Departamento de Ecologia, Universidade Federal de Goiás, Goiânia, Brazil.

${ }^{2}$ Department of Zoology, Charles University, Prague, Czech Republic.

${ }^{3}$ Departement Sciences pour l'action et le developpement, French National Institute for Agricultural Research, Paris, France.

${ }^{4}$ Staatliches Museum für Naturkunde Karlsruhe, Karlsruhe, Germany.

${ }^{5}$ Department of Ecology and Biogeography, Nicolaus Copernicus University, Toruń, Poland.

${ }^{6}$ Research Unit « Ecosystemes Biodiversite Evolution » (ECOBIO), Universite de Rennes 1 / CNRS, Rennes, France.

${ }^{7}$ Forest Ecology and Restoration Group (FORECO), Department of Life Sciences, University of Alcalá, Alcalá de Henares, Madrid, Spain.

*Corresponding author: * José Hidasi Neto, Programa de Pós-Graduação em Ecologia e Evolução, Universidade Federal de Goiás, 74001-970, Goiânia, Goiás, Brazil. Email: hidasineto@gmail.com. ORCID ID: orcid.org/0000-0003-4097-7353.

Word count (Including, Abstract and References): 6943 words

\section{ACKNOWLEDGEMENTS}

JHN thanks Paulo De Marco Jr. and Luis M. Bini for discussing the ideas presented here. JHN received a PhD scholarship by Coordernação de Aperfeiçoamento de Pessoal de Nivel Superior (CAPES) and a PhD mobility scholarship by Rennes Metropole. AMCS was supported by a Marie Curie Intra-European Fellowship (IEF 331623 'COMMSTRUCT') and a Juan de la Cierva Fellowship (IJCI-2014-19502) funded by the Spanish 'Ministerio de Economía y Competitividad'. MVC has a productivity grant awarded by CNPq (307796/2015-9). AP received a SAD fellowship from Region Bretagne. WU was supported by the institutional fund of UMK. 


\section{ABSTRACT}

Aim. An individual tree resembles a living island, a small spatially distinct unit upon which colonizers maintain populations. However, several differences exist compared to oceanic islands: a tree is relatively young, is composed of numerous differently aged branches, may be phylogenetically isolated from neighbours, and some of its colonizers are specific to particular tree lineages. We suggest that these specificities strongly affect both alpha and beta-diversity within trees, including positive effects of isolation on the diversity of generalists, and strengthening of the effect of isolation with tree age.

Location. Rennes, Bretagne, Western France

Taxon. Little-dispersive, generalist oribatid mites (Acari) and highly dispersive, specialist gall wasps (Hymenoptera: Cynipidae) on oak (Quercus sp.) trees

Methods. We tested the effects of tree and branch age, tree and branch habitat-diversity, and tree phylogenetic isolation on per-branch and per-tree alpha-diversity, and on within-tree beta-diversity of both taxonomic groups

Results. For gall wasps, no variable explained diversity patterns at any level. In contrast, for oribatid mites we found that high phylogenetic isolation of trees and high branch age increased alpha-diversity per tree and per branch (in young trees) as well as turnover among branches. High tree age decreased alpha-diversity per branch (in phylogenetically isolated trees) and increased turnover among branches. Increasing habitat diversity increased alpha-diversity per tree, but decreased alpha-diversity per branch (in young trees).

Main conclusions. For mites, contrary to common expectation, we suggest that: (i) phylogenetically distant neighbours are a source of immigration of distinct species; and (ii) with the increase of tree age, speciessorting results in a few species colonizing and dominating their preferred patches. In gall wasps, strict specialization on oaks, and efficient dispersal may render oak age or isolation unimportant. The positive relationship between isolation and within-tree turnover is a new contribution to biogeography in general.

Keywords. alpha and beta-diversity; community assembly; gall wasps; island biogeography; living island; oribatid mites; species turnover 


\section{INTRODUCTION}

Studies on oceanic islands have provided key insights into the assembly and structuring of ecological communities (Santos et al., 2016; Whittaker et al., 2017). Island properties have major effects on species diversity. Islands with higher habitat diversity typically harbour larger numbers of species because they can accommodate species with different habitat requirements (Fattorini et al., 2015; Hortal et al., 2009), particularly habitat specialists. Larger islands tend to have higher species diversity, probably because the rate of extinction relative to colonization is lower (MacArthur \& Wilson, 1967). Spatially isolated islands typically have lower species diversity, primarily because they can only be reached by few dispersers (MacArthur \& Wilson, 1967; Simberloff \& Wilson, 1969; Hendrickx et al., 2009). Finally, there is evidence that, all else being equal, young islands are occupied by less species due to little time available for their arrival (Whittaker et al., 2008; Simberloff \& Wilson, 1969; Cornell \& Harrison, 2014), although opposing forces may also operate (Whittaker et al., 2017).

Host organisms can be understood as living islands upon which entire communities, or even meta communities, of colonizers can live or feed, surrounded by an unsuitable matrix of non-host organisms (Gripenberg \& Roslin, 2005, Gossner et al., 2009; Vialatte et al., 2010; Méndez-Castro et al., 2018; Patiño et al., 2018). Many of the patterns and processes that occur on true islands can be transposed, with certain limitations, to hosts (Santos et al., 2016). Similar to oceanic islands, hosts can vary in age. We may hence hypothesize that alpha-diversity per host increases with host age, due to the accumulation of colonizers through time (albeit assembly through local speciation will not occur). Alternatively, individual species may become dominant through time, leading to a decline in species diversity (Table 1; hypotheses 1.1.1. and 1.1.2). Because hosts differ in habitat diversity, we may also hypothesize a positive relationship between colonizers' alpha-diversity and within-host habitat diversity (Table 1; hypotheses 1.2) (e.g. Gripenberg \& Roslin, 2005; Lie et al., 2009).

There are obviously many differences between oceanic islands, and living host islands. Unlike oceanic islands, phylogenetic relationships among hosts may influence the composition and diversity of colonizer species. Hosts' physical or physiological characteristics control habitat conditions available to colonizers and are often more different among distantly than among closely related host species (Revell et al., 2008). Colonizers of an individual host may perceive neighbouring hosts from distantly related species as different and unsuitable habitat. In this case, isolation should be measured as evolutionary differentiation and not as spatial distance. Therefore, for host-specialized colonizers, the phylogenetically isolated host may be surrounded by an unsuitable matrix (and may be unsuitable for specialist colonizers living on surrounding hosts) (Yguel et al., 2011, 2014). As a consequence, phylogenetically isolated hosts have been shown to harbour relatively depauperate colonizer communities (Table 1; hypothesis 1.3.1.; Vialatte et al., 2010; Yguel et al., 2014; Grandez-Rios et al., 2015). Alternatively, we hypothesize that if habitat characteristics are only moderately different among phylogenetically distant species (Revell et al., 2008; Gossner et al., 2009), or colonizers are only moderately specialized on these characteristics, phylogenetic isolation of a host may have the opposite effect. In this case, exchange among distantly related hosts remains possible and increases local species diversity due to mass effects, i.e. due to strong immigration from adjacent patches including those of 
different quality occupied by different species (Table 1; hypothesis 1.3., Brown \& Kodric-Brown, 1977; Mouquet \& Loreau, 2003; Leibold et al., 2004; Gossner et al. 2009; Table 1). To our knowledge this alternative hypothesis has not been tested so far.

Also, unlike oceanic islands, host plants are composed of numerous young patches, which can be characterized by within-patch alpha-diversity, and among-patch beta-diversity. We hypothesize that alphadiversity within these patches reflects that of the entire hosts, i.e. increases or decreases with host age or host phylogenetic isolation as explained above (Table 1; hypotheses 2.1.1. and 2.1.2). Alternatively, diversity within local patches may depend on patch characteristics rather than host characteristics. We hypothesize that alpha diversity within patches will increase with patch age due to more time available for species arrival (Table 1; hypothesis 2.2.1, Cornell \& Harrison, 2014), and with the availability of more diverse habitats for diverse specialists (Table; 1; hypothesis 2.3.1.,Hortal et al., 2009). However, according to the area-heterogeneity trade-off hypothesis (Table. 1; hypothesis 2.3.2, Kadmon \& Allouche, 2007; Allouche et al., 2012; but see Hortal et al., 2013 for criticisms), species diversity within patches decreases with habitat diversity due to a reduction in the area available per habitat, constraining, in particular, habitat specialists. To our knowledge, the importance of patch-level characters and host-level characters for patch-level alpha diversity have not been compared so far. We finally formulate hypotheses on beta-diversity among patches within hosts: it should increase with host age, because environmental heterogeneity within the hosts increases or because species differently occupying these environments arrive through time and successively fill available environments (Table 1; hypothesis 3.1). Such within-host beta-diversity may also increase with host habitat diversity because more different habitats allow the establishment of more different species communities (Table 1; hypothesis 3.2.1). Finally, we hypothesize that within host beta-diversity depends on the host's phylogenetic isolation. If host lineages strongly sort colonizer species and phylogenetic isolation impedes sorting, the few remaining colonizer species should spread across the host and, thus, reduce betadiversity among patches (Table 1; hypothesis 3.3.1.). Alternatively, if host lineages moderately sort colonizer species and phylogenetic isolation permits spill over from neighbouring host lineages, the numerous species might occur separate among patches of different environments (Table 1; hypothesis 3.3.2). To our knowledge, so far, within-host (or within-island) beta-diversity has not been studied, let alone explained, and the concept might even be new at the level of classical, oceanic islands.

Contrary to oceanic islands, the degree to which host characteristics affect the assembly of colonizer communities might increase with the strength of the co-evolutionary relationships between hosts and colonizers (Gossner et al., 2009; Yguel et al., 2014) and the degree of specialization of colonizers on particular host species (above and Castagneyrol et al., 2014). For instance, tree crowns are abundantly colonized by two groups of very small arthropods. First, oribatid mites (Acari: Oribatida) are only moderately specialized on their hosts (Behan-Pelletier \& Walter, 2000), and usually live and feed on detritus or cryptogam upon trees. They are only indirectly dependent on tree traits (e.g. bark structure) that control the accumulation of detritus or growth of cryptogams (e.g. Prinzing, 2003; Nash, 2008; for a review Walter \& Proctor, 2013). Such traits may show a moderate, albeit significant, phylogenetic signal, resulting in quantitative differences in cryptogam cover among tree lineages (Rosell et al., 2014). Second, gall wasps (Hymenoptera: Cynipidae) are highly specialized (Ambrus, 1974) to, for example, a single host genus. Indeed, gall wasps directly depend on 
host anatomical and physiological traits that affect larval development (such as sclerotization), and many of these traits show a strong phylogenetic signal (Stone et al., 2002; Hayward \& Stone, 2005). We should hence expect that phylogenetic isolation reduces diversity mostly in specialized groups such as gall wasps. In addition, different groups of colonizers also differ in dispersal capacity. Oribatid mites are flightless and depend on passive dispersal (e.g. by wind; Lehmitz et al., 2011, 2012) leading to low capacity to disperse to new hosts (Jung and Croft 2001). Overall, low dispersal capacity combined with some moderate host specialization in oribatid mites might lead to increased immigration of distinct species across short spatial distances, from phylogenetically distant neighours, through mass effect. Gall wasps, in contrast, can disperse both passively and actively across large distances (Gilioli et al., 2013). High dispersal capacity might allow finding host trees even if surrounded by phylogenetically distant neighbours.

We examine these hypotheses focusing on oribatid mites and oak gall wasps living on oak trees (Quercus spp.) in closed forest canopy. We calculate diversity measures that integrate abundances per species, as abundances provide more fine-grained information and are affected by dispersal limitation (Simberloff 2009; Boulangeat et al. 2012). We first examine the relationships between alpha-diversity of mites and gall wasps on the one hand, and microhabitat diversity, tree age, and phylogenetic isolation of the host tree on the other hand. Second, we test whether these relationships also occur at the within-tree scale of individual branches. Finally, we test whether among-branch species turnover of mite and gall wasp communities within single trees is linked to microhabitats and ages of branches, and to age and phylogenetic isolation of trees.

\section{MATERIALS AND METHODS}

\section{Selection of trees}

We sampled a temperate mixed forest located close to Rennes, Bretagne, France $(48.11 \mathrm{~N},-1.34 \mathrm{~W})$ in which oaks (Quercus petreae and Q. robur) grow surrounded by Ilex aquifolium, Fagus sylvatica, Castanea sativa, Ulmus minor, Alnus glutinosa, Sorbus torminalis, Corylus avellana, Carpinus betulus, Populus tremula, Salix caprea, Abies alba, Rhamnus frangula, Tilia cordata, Betula pendula, Prunus avium, Malus sylvestris and Pyrus pyraster. We sampled from mid-August to mid-September 2006 (Vialatte et al., 2010; Yguel et al., 2011 for details) studying nine distinct triplets - sets of three nearby (<150m) Quercus sp. trees - with each triplet composed of either Q. petreae or Q. robur; two closely related species that can hybridize (Yguel et al., 2014, note that individual trees had to be dropped from further analyses as explained below). Trees within a triplet were chosen in order to maximize differences in tree circumference at breast height and phylogenetic isolation to neighbours. Such an approach of spatially grouping ("blocking") and maximizing variation of independent variables of interest (such as age) within groups has been recommended to partial out spatially varying environmental impacts (Figure 1a; Legendre et al., 2004). Tree circumferences were used as a proxy of age (as in Vialatte et al., 2010) and ranged from 60 to $277 \mathrm{~cm}$, corresponding to 80 to 180 years old according to local forestry authorities (see Yguel et al., 2011). We did not consider younger, understory trees, as such trees are often characterized by hosting a different fauna from adult trees (Gossner et al., 2009). For 
each focal tree, phylogenetic isolation was calculated according to Vialatte et al., (2010) as $[\Sigma$ (Ntree sp. X ttree sp.)/Ntotal trees]. Ntree sp. is the number of neighbours of a particular tree species directly in contact with a focal tree's crown, tree sp. is the phylogenetic distance (in MYBP) between the establishment of the clade of the neighbouring species and the oaks, and Ntotal trees is the number of trees (all species) in contact with the focal tree's crown (Vialatte et al., 2010; Yguel et al., 2011, 2014; see Appendix S1 in Supporting Information). A tree was considered to be in contact with the focal tree when they had their leaves in contact (at least during wind), albeit barks remained separate. Phylogenetic distances were continuous (Appendix S1, Tables S1-S6). For these trees in contact we also quantified the Simpson's species diversity (as 1-D; D = $\left.\Sigma(\text { abundance of a species/total abundance })^{2}\right]$ ). We finally quantified the distance to the closest oak (either $Q$. petraea or robur, or their hybrids).

\section{Species and habitat sampling on branches and trees}

In each crown, six (for mites) and ten (for gall wasps), branches between 1.5 and 2m in length were sampled using single-rope climbing and $6 \mathrm{~m}$ branch cutters, in each of the three following strata: upper crown, lower-shaded crown, lower sun-exposed crown (Figure 1b). Branches were aged by counting back from the tip the shoot-growth branching points (identified by narrow winter growth marks). Branches were grouped into branch tips (=<6 years) and the older parts (Figure 1c; the age range of which was recorded). Each branch subsection was placed over a plastic tray and washed over its entire length with the help of a pressure washer. The solution obtained for each branch was filtered using a coffee filter, which was dipped in alcohol. Oribatid mite species (juvenile and adult individuals) were identified following Weigmann (2006). For gall wasps, we measured branch length and recorded leaf galls, excluding bud galls, identifying species based on gall morphology (Ambrus, 1974). Thus, we sampled bark for oribatids and for oribatid -specific habitat conditions, and we sampled leaves for galls and gall-specific habitat conditions, resulting in two internally consistent distinct sets of data and variables, that were analyzed separately.

We quantified alpha-diversity of gall wasps and mites per branch accounting for their abundance distributions, which is useful when overall species richness is low. Specifically, we used the unit equivalent of Simpson's diversity [calculated as 1-D; $D=\sum$ (abundance of a species/total abundance $)^{2}$ ], using abundances per branch. Simpson's index is largely independent of sample size (Rosenzweig 1995), contrary to species numbers which would need to be rarified. To avoid potential under-sampling and zero-inflated data we only considered branches and trees with Simpson's diversity $>0$. Alpha-diversity for the entire tree was calculated as the Simpson's diversity (1-D) of the averaged abundances of each species on the entire tree ("per-tree alpha-diversity"). Mite communities were strongly dominated by two species. Nevertheless high alpha-diversity did not simply reflect these two species to have similar abundances (Appendix S1).

We also calculated within-tree turnover and nestedness-components of beta-diversity (Baselga, 2010) using averaged values between each pair of branches of a tree based on a Bray-Curtis distance of the occurrences of mites ('bray.part' function from 'betapart' package; Baselga \& Orme, 2012). Our measure of turnover is particularly unbiased by unequal sampling efforts resulting from unequal numbers of animals per sample and particularly independent of nestedness (Barwell et al., 2015). We used pairwise turnover and 
nestedness values to calculate average turnover and nestedness for each tree. But, we note that our hypotheses refer to turnover.

Oribatid mites are usually associated with algae, fungi, moss or lichens, commonly feeding on these organisms (Walter \& Proctor, 2013, Prinzing, 2003). Consequently, the distribution of oribatids should depend on the presence of these microhabitats. Therefore, we measured the coverage (\%) per branch of algae, mosses, crustose lichens, foliose lichens, and "mixed" (intermingled cryptogams). Again, we used the Simpson's metric to assess habitat diversity per branch and per tree. Habitat diversity per branch was calculated as the Simpson's diversity of habitat measurements on the branch. Habitat diversity per tree was calculated as the Simpson's diversity of the averaged per-branch measurements of each habitat variable. To measure habitat composition and reduce data dimensionality, we also used the scaled and centered percentages of each habitat variable to perform a Principal Components Analysis (PCA) using the KaiserGuttman criterion for axis selection ('vegan' package in R; Dixon, 2003).

Gall wasp larvae feed on plant tissue. Consequently, the distribution of gall wasps should depend on the composition of plant tissues. To identify habitat conditions of gall wasp larvae, we sampled ten leaves from each stratum (upper crown, lower shaded crown, and lower exposed crown) of each tree (always the third leaf from base of the branch) and cooled them until chemical analysis. We measured leaf $\mathrm{C} / \mathrm{N}$ and Dry mass contents according to standard protocols as detailed in Appendix S1. Habitat diversity of a tree was then calculated as the average of standard deviations of $\mathrm{C} / \mathrm{N}$ and dry mass. Note that such diversity of leaves as habitats was not available at branch-scale contrary to the cryptogam measures.

\section{Explaining diversity}

We tested the hypotheses summarized in Table 1. In short, we statistically explained alpha-diversity per tree as well as alpha-diversity per branch and beta-diversity per tree among branches by: ages, habitat diversities and phylogenetic isolations and their interactions. For per-tree data we used ordinary multiple regression analyses. For the more complex per-branch data nested within trees, we used mixed-effect regression enriched by model averaging procedures. Variables were log transformed prior to the statistical analyses, except for principal component axes. Independent variables were weakly correlated (unsigned relationships mostly < 0.4; Appendix S1, Tables S3 and S6), indicating that multicollinearity was not an issue, consistent with the high adjusted $R^{2}$ of our analyses. We also conducted a preliminary analysis to explore the role of variables not accounted for by the hypotheses: spatial autocorrelation among studied trees, spatial distance to the closest oak tree and species diversity of the ambient canopy. We found that these variables do not relate to alpha diversities (Appendix S1).

\section{Alpha-diversity per tree}

We tested the effects of tree crown's age, its habitat diversity, and its phylogenetic isolation on the tree crown's alpha-diversity of mites and gall wasps. For both, we fitted independent multiple regressions using Simpson's diversity per tree as a response variable, and tree circumference, tree phylogenetic isolation, and 
habitat diversity per tree as predictor variables. To account for possible changes in the effects of each predictor variable at different values of the other predictors, we fitted three further models, each including one of the following interactions: tree age : habitat diversity per tree, tree phylogenetic isolation : habitat diversity per tree, and phylogenetic isolation : tree age (including all interaction terms together would lead to excessive multicollinearity). We then chose the model with the lowest value of sample-size corrected Akaike's Information Criteria (AICC) (Bunnefeld \& Phillimore, 2012). In the mite dataset, after evaluating the residuals (using probability and predicted-vs-residual plots), we removed a maximum of three outliers in order to provide a better fit to our model. This procedure did not qualitatively change results, but adjusted $R^{2}$ increased from 0.294 to 0.689 .

\section{Explaining alpha-diversity per branch}

We fitted mixed-effects regressions (Bunnefeld \& Phillimore, 2012) to analyze how characteristics of trees and branches explain species diversity at the within-tree scale. We used colonizer species Simpson's diversity per branch as the response variable, and remind here that this variable is different from tree-level alpha-diversity analyzed above: as tree-level diversity reflects the combined effect of branch-level diversity and turnover among branches. As predictor variables we used tree age, tree phylogenetic-isolation, and either branch age (for mites) or branch length (for gall wasps). Trees where the branches were collected were used as random effects. As above, we also fitted three other models with the same variables, but including either of the following interactions: tree age : branch age or length, tree phylogenetic isolation : branch age or length, and tree phylogenetic isolation : tree age. We chose the models with the lowest values of AICc. To account for the uncertainty in the selection of sets of variables in mixed effects models, we then conducted a modelaveraging procedure. For both mites and gall wasps' models, we permuted the fixed-effect variables found in the best mixed-effects model, fitting a new mixed-effects model for each subset ('dredge' function; Bunnefeld \& Phillimore, 2012; Bartoń, 2015). Next, we generated averaged models for mites and gall wasps using subset models with $\triangle \mathrm{AICc}<2$ ('model.avg' function from 'MuMIn' R package; R version 3.4.2; R Core Team, 2017; Burnham \& Anderson, 2002; Bartoń, 2015). Importance values were calculated for each predictor variable as the sum of Akaike weights of all models in which the variable appeared (Burnham \& Anderson, 2002). After this procedure, mites had only one model with delta $A I C c<2$, so we interpreted results from this mixed-model. Also, for mites we repeated this approach including as explanatory variables the log of habitat diversity per branch and three axes of the PCA performed with the habitat variables (related to, respectively, presence of uncovered branch and lack of algae; lack of crustose lichens and mosses; and the presence of foliose lichen (Appendix S1, Tables S7-S9; equivalent per-branch data was not available for galls). In this analysis we also included two additional interactions: tree age : habitat diversity per branch, and tree phylogenetic isolation : habitat diversity per branch. We calculated averaged models again using the threshold of delta $A I C c<2$. We only discuss results from this latter analysis for mites as it is the most complete. In all model-averaging procedures, we included the marginal and conditional $R^{2}$ of subset models. These values represent, respectively, the variance explained by only fixed and by fixed and random variables (calculated with 'r.squaredGLMM' function; Nakagawa \& Schielzeth, 2013). 
We performed multiple linear regressions using as response variables the average turnover and nestedness partitions of beta-diversity among branches of trees. We used tree age, tree phylogenetic isolation, and habitat diversity per tree as predictor variables. We again tested for interactions by fitting models including the following interactions: tree age : habitat diversity per tree, tree phylogenetic isolation : habitat diversity per tree, and tree phylogenetic isolation : tree age. We chose models with the lowest AICc values. For the mites dataset, after evaluating the residuals, we decided to remove three outliers (not the same as mentioned previously) for the model using turnover as a response variable. This procedure did not qualitatively change results, but adjusted $R^{2}$ increased from 0.53 to 0.66 . Statistical representations of interaction effects were visualized using 'visreg' R package (Breheny \& Burchett, 2015).

\section{RESULTS}

A total of 25 mite species (including one undetermined) and 10 gall wasp species were recorded on 181 branches from 25 trees and 153 branches from 21 trees (Appendix S2, Tables S10 and S11). Mite abundance varied from 40 to 1028 individuals per tree, with Micreremus brevipes (Michael, 1888) being the most abundant. Gall wasp abundance varied from 6 to 414 individuals per tree, and Neuroterus anthracinus (Curtis, 1838) was the most abundant species.

\section{Alpha-diversity per tree}

For both mites and gall wasps, the best AICc model included no interactions (Table 2 throughout; for galls Appendix S3). For mites, alpha-diversity increased with both increasing phylogenetic isolation (Figure $2 a$ ) and tree-level habitat diversity (Figure $2 b$ ). Tree age did not explain mite alpha-diversity. In the case of gall wasps, the best AICc model did not explain variation in alpha-diversity $\left(F(3,17)=1.098\right.$; adjusted $R^{2}=0.014$; $\mathrm{p}=0.377$, Appendix S3).

\section{Alpha-diversity per branch}

For gall wasps, the best AICc model was the one with no interaction (Appendix S3). However, no predictor was significant, explained variance was extremely low and none of the relationships was significant (Table 3). For mites, the best AICc model included the interaction between phylogenetic isolation and tree age (Table 2). In the additional model including information on branch habitat composition (PCA scores), the best model included the interaction between tree age and branch habitat diversity (Appendix S3). When per-branch habitat composition variables were not considered, mite diversity increased with phylogenetic isolation and tree age on single branches. The significant interaction term between phylogenetic isolation and age indicated that branches of more phylogenetically isolated trees had higher mite diversity when the tree was young, but lower diversity when the tree was old (Table 1; Appendix S1, Figure S1). When including habitat composition variables (Table 3), phylogenetic isolation increased mite diversity, while tree age and habitat diversity per branch decreased mite diversity. The significant interaction between habitat diversity per branch and tree age 
indicated that high habitat diversity decreased mite diversity in young and increased diversity in old trees (Appendix S1, Figure S2). Branch age was in all models positively related to mite diversity (Table 3; Figure 2c).

\section{Beta-diversity among branches}

For gall wasps, no interaction was included (Appendix S3), no predictor was significant and explained variance was extremely low (both adjusted $R^{2}<0.05, p>0.5$; Appendix S3). The best AICc model with average turnover as a response variable was the one that included the interaction between phylogenetic isolation and tree age for mites (Table 2). Specifically, for mites, turnover was higher in trees with high habitat diversity and phylogenetic isolation (Table 2; Figure 2d). The significant interaction between tree age and tree phylogenetic isolation indicated that mite turnover increased with tree age in phylogenetically non-isolated trees, while decreased in isolated trees (Table 2; Appendix S1: Figure S3). The best AICc model with average nestedness as a response variable did not contain this interaction between tree age and tree habitat diversity for mites (Table 2; Appendix S3). Specifically, mite nestedness was higher in old than in young trees (Table 2). Also, mite nestedness increased with tree age when trees provided low habitat diversity, but decreased when trees were habitat diverse (Table 2; Appendix S1: Figure S4).

\section{DISCUSSION}

We characterized host trees as particular, living islands. We found that tree age, phylogenetic isolation, and habitat diversity control alpha-diversity of colonizers on the entire living islands, as well as on and among their individual branches (while other neighbourhood-related characteristics were not significant; Appendix S1). Notably, such patterns only occurred for oribatid mites, which have poor dispersal ability and are less specialized compared with gall wasps.

We found higher mite alpha-diversity on phylogenetically isolated host trees across the entire trees and on each of their branches;This result is consistent with hypothesis 1.3.2, but contradicts 1.3.1. and what has been observed before (reviewed by Grandez-Rios et al., 2015 for species richness as a measure of alphadiversity). We suggest that mite populations represent a different evolutionary situation from that found in other taxa studied before. Mites do not feed on the tree itself and hence do not directly depend on tree traits nor on their phylogenetic signal. However, mites depend indirectly on some tree traits, such as bark structure and $\mathrm{pH}$, that control cryptogam cover (Nash, 2008) and thus substrate and food of oribatids. Such bark traits appear to show moderate but significant phylogenetic signal (e.g. Rosell et al., 2014), resulting in differences in cryptogam covers among tree lineages. Consequently, distantly related trees should be preferred by different mite species, while most mites could be able to survive on most tree species, even if they present lower habitat quality for some of them (Behan-Pelletier \& Walter, 2000 for a review). Arboreal mites are frequently dispersed passively through wind (Lehmitz et al., 2011; Lehmitz et al., 2012) and a host surrounded by distant relatives might hence be colonized by the mites preferring neighbouring hosts. Consequently, species diversity should increase on evolutionarily isolated trees due to mass effects. The idea that mass effects may contribute to local species richness under particular conditions is not new itself (Mouquet \& Loreau, 2003). Nevertheless, here we found evidence that for colonizers of hosts, such mass 
effects can be particularly prominent in phylogenetically diverse host communities, and under intermediate (or indirectly operating) phylogenetic signal of traits controlling the habitat quality of such hosts.

Two relationships were opposite at tree and at branch level. First, we found a positive relationship between habitat diversity and mite alpha-diversity per tree, but a negative relationship per branch (consistent with hypotheses 1.2.1.and 2.3.2, respectively, Table 1). An increase of species alpha diversity with habitat diversity is intuitive as habitat specialists can better accommodate themselves at islands with a high variation of resources and conditions available (Fattorini et al., 2015; Hortal et al., 2009). A decrease of species diversity on braches with high habitat diversity, in contrast, might reflect the decreased area available for a given suitable habitat. Second, we found that mite alpha-diversity per branch decreased with host-tree age (at least when branches had low habitat diversity), but increased with branch age (consistent with hypotheses 2.1.2. and 2.2.1., respectively, Table 1). Older trees might increase the chance that those species that are more efficient in colonizing a specific tree will dominate all branches of the crown sometime after arrival (Badano et al., 2005; Lekevicius, 2009, for 'classical' islands). Overall, the local communities within, and the species pool across a given host individual appear to be driven by opposing effects, in part perhaps because the local communities are confined to very small modules (branches) where habitat surface might become a limiting factor, and their filling by species takes time.

Tree characteristics influenced spatial turnover of mites among branches within trees. Precisely, mite turnover increased with phylogenetic isolation (consistent with hypothesis 3.3.2., Table 1). A possible explanation invokes again mass effects from phylogenetically distant neighbours that increase the pool of species that colonize different branches (Badano et al., 2005; Lekevicius, 2009, for 'classical' islands). Further, tree age gives time for habitat filtering by sorting specialists into their most suitable microhabitats (tree branches) (consistent with hypothesis 3.2., Table 1). We are not aware of any study reporting the effect of isolation and age of hosts or islands on the assembly of communities among patches within these hosts or islands. There are, however, studies that describe the assembly within and among communities based on the properties of landscape mosaics in which these communities are embedded (Hendrickx et al., 2009; Chisholm et al., 2011). Assembly processes on individual hosts may hence be captured by concepts of landscape ecology, albeit landscape ecologists have so far not accounted for the effect of the age of an entire landscape or the degree of its isolation from other landscapes.

Contrary to the findings regarding oribatids, the assembly of gall wasps does not seem to be driven by the characteristics of hosts we have evaluated here (Table 1). Contrary to oribatids, gall wasps are good dispersers that present high host-specialization and that can even alter their habitats by inducing the growth of plant tissue (Stone et al., 2002; Hayward \& Stone, 2005). These contrasting ecological characters might at least partly explain why gall wasps are less dependent on characters of their living islands and their neighbourhoods than mites (Figure 3). The fact that species from both ecological groups were sampled on the same trees, and with sample sizes in similar orders of magnitude, strengthens our confidence that the observed differences do not stem from methodological biases. Based on the same experiment as the present study, Yguel et al. (2011, 2014) found that the alpha-diversity of chewing phytophages (mainly Lepidoptera) was lower on phylogenetically isolated host trees. Notably, lepidopterans have intermediate (between oribatids 
and gall wasps) degrees of host-specialization and dispersal capacities (for Lepidoptera in the present study system; Yguel et al., 2011). Therefore, lepidopterans on phylogenetically distant neighbours may be less capable of colonizing a focal tree than are oribatid mites, and lepidopterans from spatially distant but phylogenetically proximate host trees might be less capable of finding focal trees than are gall wasps (Figure 3). Similar effects of host phylogenetic isolation that was found for gall wasps might also occur in chalcidoid wasps (Yguel et al., 2014). Overall, a given character of a host - being surrounded by distantly related neighbours - may have opposite effects on colonizer organisms that differ in host specialization and dispersal ability. We stress however that this conclusion resides on a single taxon per type of colonizer biology. Further confirmations for other taxa are needed.

We are aware that our study may present some limitations. Sampling was done during the end of summer, so identified patterns might not fully reflect the effects of tree characteristics on the assembly of colonizers. This is especially important for gall wasps, which might produce smaller generations and smaller galls during spring than in summer (Hayward \& Stone, 2005). Also, sampling was restricted to peripheral branches, leaving out major branches, deadwood and trunks, with their deeply fissured bark and thick, threedimensional cryptogam cover harbouring different species of oribatid mites in high abundances. We hence do not pretend to characterize the entire oribatid fauna of a tree, but only that of one relatively young structure, comparable among trees of all ages. Finally, our sample size is limited, albeit this would not explain any observed significant effects. Moreover, our major results are relatively solid (e.g. with non-significant effects with $\mathrm{p}>0.05$, adjusted $R^{2}$ up to 0.689 , and partly based on averaging across numerous models; see Table 2 ), which increases our confidence in the conclusions taken from them.

Our results suggest that, unless colonizers can easily reach and manipulate host trees, forest trees can function as living islands. However, these living islands present specificities, such as phylogenetic isolation from neighbours and rapid growth of individual patches, which can in turn determine the assembly of their colonizers. Phylogenetically isolated trees may, for the studied mites, have increased diversity consistent with mass effects from distantly related neighbour hosts, while old trees have decreased colonizer diversity within patches, consistent with sorting of organisms into their preferable patches through time. Distinct metacommunity perspectives (mass effects and species sorting) may explain how trees as living islands influence the assembly of their colonizers. While assembly may be driven by isolation, age and habitat diversity as on true islands, the processes invoked here are partly different from those on true islands. Specifically, we suggest that the level of insularity is controlled also by the biology of the colonizers themselves, insularity being highest for colonizers that disperse little and depend directly on host traits that show strong phylogenetic signal. 


\section{REFERENCES}

Allouche, O., Kalyuzhny, M., Moreno-Rueda, G., Pizarro, M., \& Kadmon, R. (2012) Area-heterogeneity tradeoff and the diversity of ecological communities. Proceedings of the National Academy of Sciences, 109, 17495-17500.

Ambrus, B. (1974) Cynipida-Gubacsok-Cecidia Cynipidarum. Fauna Hungariae, Hymenoptera II, vol 12, part 1/a pp. 119. Akadémiai Kiadó, Budapest.

Badano, E.I., Regidor, H.A., Núñez, H.A., Acosta, R., \& Gianoli, E. (2005) Species richness and structure of ant communities in a dynamic archipelago: Effects of island area and age. Journal of Biogeography, 32, 221-227.

Bartoń, K. (2015) MuMIn: Multi-model inference. R package version 1.15.1. https://cran.rproject.org/web/packages/MuMIn/MuMIn.pdf.

Barwell, L.J., Isaac, N.J.B., \& Kunin, W.E. (2015) Measuring $\beta$-diversity with species abundance data. Journal of Animal Ecology, 84, 1112-1122.

Baselga, A. (2010) Partitioning the turnover and nestedness components of beta-diversity. Global Ecology and Biogeography, 19, 134-143.

Baselga, A. (2013) Separating the two components of abundance-based dissimilarity: balanced changes in abundance vs. abundance gradients. Methods in Ecology and Evolution, 4, 552-557.

Baselga, A. \& Orme, C.D.L. (2012) betapart: an R package for the study of beta-diversity. Methods in Ecology and Evolution, 3, 808-812.

Behan-Pelletier, V. \& Walter, D.E. (2000) Biodiversity of oribatid mites (Acari: Oribatida) in tree canopies and litter. Invertebrates as webmasters in ecosystems (ed. by D.C. Coleman and P.F. Hendrix), pp. 187-202. CABI, New York.

Breheny, P. \& Burchett, W. (2015) visreg: Visualization of Regression Models. R package version 2.2. https://cran.r-project.org/web/packages/visreg/visreg.pdf.

Bunnefeld, N. \& Phillimore, A.B. (2012) Island, archipelago and taxon effects: mixed models as a means of dealing with the imperfect design of nature's experiments. Ecography, 35, 15-22.

Burnham, K.P. \& Anderson, D.R. (2002) Model Selection and Multimodel Inference: A Practical InformationTheoretic Approach. Springer-Verlag, New York.

Castagneyrol, B., Jactel, H., Vacher, C., Brockerhoff, E.G., \& Koricheva, J. (2014) Effects of plant phylogenetic diversity on herbivory depend on herbivore specialization. Journal of Applied Ecology, 51, 
465 Chisholm, C., Lindo, Z., \& Gonzalez, A. (2011) Metacommunity diversity depends on connectivity and patch arrangement in heterogeneous habitat networks. Ecography, 34, 415-424.

Cornell, H. V \& Harrison, S.P. (2014) What are species pools and when are they important? Annual Review of Ecology, Evolution, and Systematics, 45, 45-67.

Denno, R.F., McClure, M.S., \& Ott, J.R. (1995) Interspecific interactions in phytophagous insects: competition reexamined and resurrected. Annual Review of Entomology, 40, 297-331.

Dixon, P. (2003) A package of R functions for community ecology. Journal of Vegetation Science, 14, 927930.

Fattorini, S., Dapporto, L., Strona, G., \& Borges, P.A.V. (2015) Calling for a new strategy to measure environmental (habitat) diversity in Island Biogeography: a case study of Mediterranean tenebrionids (Coleoptera: Tenebrionidae). Fragmenta Entomologica, 47, 1-14.

Gilioli, G., Pasquali, S., Tramontini, S., \& Riolo, F. (2013) Modelling local and long-distance dispersal of invasive chestnut gall wasp in Europe. Ecological Modelling, 263, 281-290.

Gossner, M.M., Chao, A., Bailey, R.I., \& Prinzing, A. (2009) Native fauna on exotic trees: phylogenetic conservatism and geographic contingency in two lineages of phytophages on two lineages of trees. The American Naturalist, 173, 599-614.

Grandez-Rios, J.M., Bergamini, L.L., De Araujo, W.S., Villalobos, F., \& Almeida-Neto, M. (2015) The effect of host-plant Phylogenetic isolation on species richness, composition and specialization of insect herbivores: A comparison between native and exotic hosts. PLOS ONE, 10, e0138031.

Gripenberg, S. \& Roslin, T. (2005) Host plants as islands: Resource quality and spatial setting as determinants of insect distribution. Annales Zoologici Fennici, 42, 335-345.

Hayward, A. \& Stone, G.N. (2005) Oak gall wasp communities: Evolution and ecology. Basic and Applied Ecology, 6, 435-443.

Hendrickx, F., Maelfait, J.P., Desender, K., Aviron, S., Bailey, D., Diekotter, T., Lens, L., Liira, J., Schweiger, O., Speelmans, M., Vandomme, V., \& Bugter, R. (2009) Pervasive effects of dispersal limitation on within- and among-community species richness in agricultural landscapes. Global Ecology and Biogeography, 18, 607-616.

Hortal, J., Triantis, K.A., Meiri, S., Thébault, E., \& Sfenthourakis, S. (2009) Island species richness increases 
with habitat diversity. The American Naturalist, 174, E205-E217.

Legendre, P., Dale, M.R.T., Fortin, M., Casgrain, R., \& Gurevitch, J. (2004) Effects of spatial structures on the results of field experiments. Ecology, 85, 3202-3214.

Lehmitz, R., Russell, D., Hohberg, K., Christian, A., \& Xylander, W.E.R. (2011) Wind dispersal of oribatid mites as a mode of migration. Pedobiologia, 54, 201-207.

Lehmitz, R., Russell, D., Hohberg, K., Christian, A., \& Xylander, W.E.R. (2012) Active dispersal of oribatid mites into young soils. Applied Soil Ecology, 55, 10-19.

Lekevicius, E. (2009) Vacant niches in nature, ecology, and evolutionary theory: a mini-review. Ekologija, 55, 165-174.

Lie, M.H., Arup, U., Grytnes, J.-A., \& Ohlson, M. (2009) The importance of host tree age, size and growth rate as determinants of epiphytic lichen diversity in boreal spruce forests. Biodiversity and Conservation, 18, 3579-3596.

MacArthur, R.H. \& Wilson, E.O. (1967) The Theory of Island Biogeography. Princeton University Press, Princeton, New Jersey.

Méndez-Castro, F.E., Bader, M.Y., Mendieta-Leiva, G. \& Rao, D. (in press) Islands in the trees: A biogeographic exploration of epiphyte-dwelling spiders. Journal of Biogeography.

Mouquet, N. \& Loreau, M. (2003) Community patterns in source-sink metacommunities. The American Naturalist, 162, 544-557.

Nakagawa, S. \& Schielzeth, H. (2013) A general and simple method for obtaining $\mathrm{R}^{2}$ from generalized linear mixed-effects models. Methods in Ecology and Evolution, 4, 133-142.

Nash, T.H. (2008) Lichen Biology. Cambridge University Press, Cambridge.

Patiño J., Gómez-Rodríguez C., Pupo-Correia A., Sequeira M. \& Vanderpoorten A. (2018) Trees as habitat islands: Temporal variation in alpha and beta-diversity in epiphytic laurel forest bryophyte communities. Journal of Biogeography, in press

Prinzing, A. (2003) Are generalists pressed for time? An interspecific test of the Time-Limited Disperser Model. Ecology, 84, 1744-1755.

R Core Team. (2017). R: A language and environment for statistical computing. Vienna, Austria: R Foundation for Statistical Computing. Retrieved from http://www.R-project.org/.

Revell, L.J., Harmon, L.J., \& Collar, D.C. (2008) Phylogenetic signal, evolutionary process, and rate. 
Systematic Biology, 57, 591-601.

Rosell, J.A., Gleason, S., Méndez-Alonzo, R., Chang, Y., \& Westoby, M. (2014) Bark functional ecology: evidence for tradeoffs, functional coordination, and environment producing bark diversity. New Phytologist, 201, 486-497.

Rosenzweig, M.L. (1995) Species Diversity in Space and Time. Cambridge University Press.

Santos A.M.C., Field R. \& Ricklefs R.E. (2016) New directions in island biogeography. Global Ecology and Biogeography, 25, 751-768

Stone, G.N., Schönrogge, K., Atkinson, R.J., Bellido, D., \& Pujade-Villar, J. (2002) The population biology of oak gall wasps (Hymenoptera: Cynipidae). Annual Review of Entomology, 47, 633-668.

Vialatte, A., Bailey, R.I., Vasseur, C., Matocq, A., Gossner, M.M., Everhart, D., Vitrac, X., Belhadj, A., Ernoult, A., \& Prinzing, A. (2010) Phylogenetic isolation of host trees affects assembly of local Heteroptera communities. Proceedings of the Royal Society B, 277, 2227-2236.

Walter, D.E. \& Proctor, H.C. (2013) Mites: Ecology, Evolution \& Behaviour. Springer, New York.

Weigmann, G. (2006) Hornmilben (Oribatida). Die Tierwelt Deutschlands 76 (ed. by F. Dahl), pp. 520. Goecke \& Evers, Keltern.

Whittaker, R.J., Triantis, K.A. \& Ladle, R.J. (2008) A general dynamic theory of oceanic island biogeography. Journal of Biogeography, 35, 977-994.

Whittaker R.J., Fernández-Palacios J.M., Matthews T.J., Borregaard M.K. \& Triantis K.A. (2017) Island biogeography: Taking the long view of nature's laboratories. Science, 357, eaam8326.

Yguel, B., Bailey, R., Tosh, N.D., Vialatte, A., Vasseur, C., Vitrac, X., Jean, F., \& Prinzing, A. (2011) Phytophagy on phylogenetically isolated trees: Why hosts should escape their relatives. Ecology Letters, 14, 1117-1124.

Yguel, B., Bailey, R.I., Villemant, C., Brault, A., Jactel, H., \& Prinzing, A. (2014) Insect herbivores should follow plants escaping their relatives. Oecologia, 521-532. 
550 Table 1. Summary of studied alternative hypotheses on the effects of host-tree and branch characteristics on colonizer faunas, in a spatially 551 contiguous forest canopy of adult, similar sized trees. The "+" and "-" mean, respectively, expected positive or negative relationships between predictor and response variables. Hypotheses corroborated for oribatids are in bold. No hypothesis was corroborated for gall wasps.

$\begin{array}{llll}\text { Response variable } & \text { Predictor variable } & \text { Alternative Hypothesis 1 } & \text { Alternative Hypothesis } 2\end{array}$

\begin{tabular}{|c|c|c|}
\hline 1.1. & $\begin{array}{l}\text { Alpha-diversity per } \\
\text { host }\end{array}$ & Age of host \\
\hline 1.2 . & & Habitat diversity of host \\
\hline 1.3. & & $\begin{array}{l}\text { Host phylogenetic } \\
\text { isolation of host }\end{array}$ \\
\hline
\end{tabular}
2.1. Alpha-diversity per patch within host
Age and phylogenetic isolation of hosts
2.2 .
Patch age and its interaction with host characteristics
2.3 Habitat diversity per patch and its interaction with host characteristics
3.1. Within-host beta- Age of host diversity
Habitat diversity of host
3.2 .
+ age and phylogenetic isolation may increase species richness in the hosts (explained above) and hence in its constituent patches.
+ more time available for the arrival of species in older patches within hosts - provided that host age and phylogenetic isolation ensure a large species pool.
+ more habitats will be available for species with different niches - provided that host age and phylogenetic isolation ensure a large species pool.

\section{+ because environmental heterogeneity within hosts} increases or because species differently occupying these environments arrive through time.
+ more habitats will be available for different species with different niches.
Phylogenetic isolation of - if host lineages strongly sort colonizer species and host phylogenetic isolation impedes sorting, the few remaining colonizer species may spread across the host and reduce turnover among patches

+ the older the host the more likely a colonization occurred.

+ the more diverse the habitats the larger the niche space.

- if relevant host characteristics for colonizers are

phylogenetically strongly conserved or colonizers are highly specialized. Then isolated hosts will be hard to reach for the species that can live there (ecological sorting, impeded by isolation).

- the older the host the more likely it is dominated by particular species.

None.

+ if relevant host characteristics for colonizers are phylogenetically moderately conserved or colonizers are only moderately specialized, then isolated hosts will receive species from neighbouring, phylogenetically distant hosts (mass effect, facilitated by isolation).

- age and phylogenetic isolation may decrease species diversity in the host (explained above) and hence in its constituent patches.

None.

- the area available per habitat will also decrease, constraining in particular habitat specialists. None. 
Table 2. Effects of age and phylogenetic isolation on alpha-diversity, turnover and nestedness of mites in crowns of host trees (using multiple regression analysis with best subset search based on delta AICc<2). Alpha-diversity response variables were (1) tree-level species Simpson's diversity $[F(3,20)=16.54$; adjusted $R^{2}=0.689 ; p$-value $<0.001$ ], and (2) branch-level species Simpson's diversity (observations=181; groups=25; Marginal $R^{2}=0.152$; Conditional $R^{2}=0.201$; see Table 2 for analyses including habitat covariables). Betadiversity response variables were: (3) average mite turnover $\left[F(4,17)=11.19\right.$; adjusted $R^{2}=0.66$; $p$ value $<0.001]$, and (4) average mite nestedness $\left[F(4,20)=3.619\right.$; adjusted $R^{2}=0.304 ; p$-value $\left.=0.022\right]$ among branches of each tree. Significant $p$-values are in bold. Equivalent analyses for gall wasps were all nonsignificant with $\mathrm{R}^{2}<0.015$. Variables were In+1-transformed, except for $\mathrm{PC}$ axes.

\begin{tabular}{|c|c|c|c|c|}
\hline Predictor variables & Estimate & SE & t value & p-value \\
\hline \multicolumn{5}{|l|}{ PER-TREE MITE ALPHA-DIVERSITY } \\
\hline (Intercept) & -0.362 & 0.120 & -3.025 & 0.007 \\
\hline phylogenetic isolation & 0.093 & 0.015 & 6.322 & $<0.001$ \\
\hline tree age & -0.005 & 0.042 & -0.123 & 0.904 \\
\hline tree habitat diversity & 1.055 & 0.211 & 4.999 & $<0.001$ \\
\hline \multicolumn{5}{|l|}{ PER-BRANCH MITE ALPHA-DIVERSITY } \\
\hline (Intercept) & -0.587 & 0.236 & -2.487 & 0.014 \\
\hline tree age & 0.877 & 0.315 & 2.78 & 0.011 \\
\hline phylogenetic isolation & 0.211 & 0.063 & 3.324 & 0.003 \\
\hline branch age & 0.076 & 0.025 & 3.055 & 0.003 \\
\hline phylogenetic isolation: tree age & -0.241 & 0.091 & -2.648 & 0.015 \\
\hline \multicolumn{5}{|c|}{ WITHIN-TREE MITE TURNOVER AMONG BRANCHES } \\
\hline (Intercept) & -1.326 & 0.222 & -5.963 & $<0.001$ \\
\hline $\log ($ phylogenetic isolation +1$)$ & 0.366 & 0.063 & 5.811 & $<0.001$ \\
\hline $\log ($ tree age +1$)$ & 1.645 & 0.300 & 5.483 & $<0.001$ \\
\hline $\log$ (tree habitat diversity +1 ) & 0.577 & 0.181 & 3.182 & 0.005 \\
\hline $\log ($ phylogenetic isolation +1$): \log ($ tree age +1$)$ & -0.497 & 0.090 & -5.498 & $<0.001$ \\
\hline \multicolumn{5}{|c|}{ WITHIN-TREE MITE NESTEDNESS AMONG BRANCHES } \\
\hline (Intercept) & -0.260 & 0.386 & -0.673 & 0.509 \\
\hline phylogenetic isolation & 0.010 & 0.017 & 0.603 & 0.554 \\
\hline tree age & 0.871 & 0.383 & 2.275 & 0.034 \\
\hline tree habitat diversity & 1.301 & 0.817 & 1.593 & 0.127 \\
\hline tree age: tree habitat diversity & -2.055 & 0.863 & -2.381 & 0.027 \\
\hline
\end{tabular}


Table 3. Effects of habitat variables, and (as in Table 2) age and phylogenetic isolation on Simpson's alpha-diversity of mites and gall wasps in crowns of host trees. Averaged models of parameter estimates, each based on five models (averaged marginal $R^{2}=0.166$; averaged conditional $R^{2}=0.220$ ), and three (averaged marginal $R^{2}=0.002$; averaged conditional $R^{2}=0.131$ ) subset models. Variables were In+1transformed, except for PC axes.

\begin{tabular}{lcccccc}
\hline & & \multicolumn{4}{c}{ Adjusted } \\
Predictor variable & Importance & Estimate & SE & SE & z value & p-value \\
\hline PER-BRANCH MITE ALPHA-DIVERSITY & WITH PER-BRANCH HABITAT VARIABLES & & \\
(Intercept) & & 0.256 & 0.13 & 0.131 & 1.957 & $\mathbf{0 . 0 5}$ \\
phylogenetic isolation & 1 & 0.042 & 0.014 & 0.015 & 2.808 & $\mathbf{0 . 0 0 5}$ \\
branch age & 1 & 0.067 & 0.026 & 0.026 & 2.57 & $\mathbf{0 . 0 1}$ \\
tree age & 1 & -0.251 & 0.114 & 0.12 & 2.089 & $\mathbf{0 . 0 3 7}$ \\
branch habitat diversity & 1 & -0.733 & 0.25 & 0.252 & 2.91 & $\mathbf{0 . 0 0 4}$ \\
tree age:branch habitat diversity & 1 & 0.9 & 0.314 & 0.317 & 2.841 & $\mathbf{0 . 0 0 5}$ \\
PC2 & 0.59 & -0.007 & 0.008 & 0.008 & 0.833 & 0.405 \\
PC3 & 0.29 & 0.003 & 0.007 & 0.007 & 0.423 & 0.673 \\
PC1 & 0.12 & $<0.001$ & 0.003 & 0.003 & 0.136 & 0.892 \\
PER-BRANCH GALL WASP ALPHA-DIVERSITY & & & & & \\
(Intercept) & & 0.322 & 0.041 & 0.042 & 7.712 & $<\mathbf{0 . 0 0 1}$ \\
tree age & & 0.008 & 0.026 & 0.028 & 0.296 & 0.767 \\
branch length & 0.248 & $<0.001$ & 0.006 & 0.006 & 0.156 & 0.876 \\
\hline
\end{tabular}




\section{FIGURES}

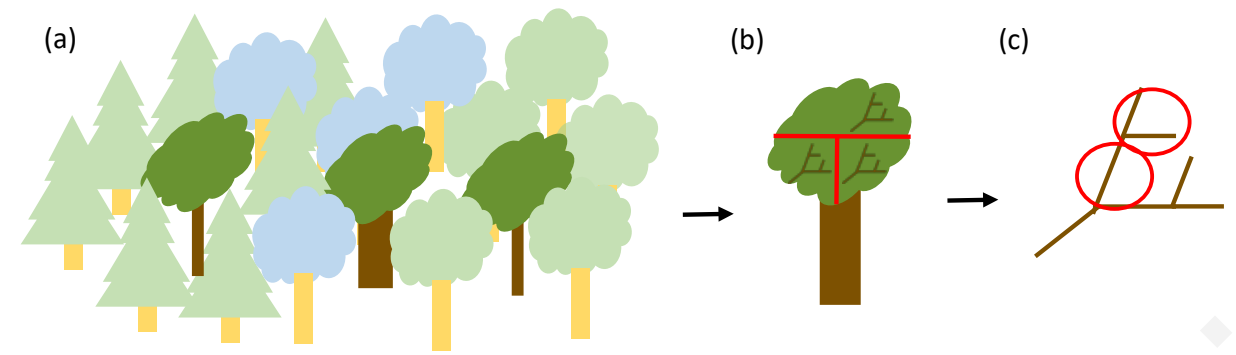

Figure 1. 
$\frac{n}{\pi}$

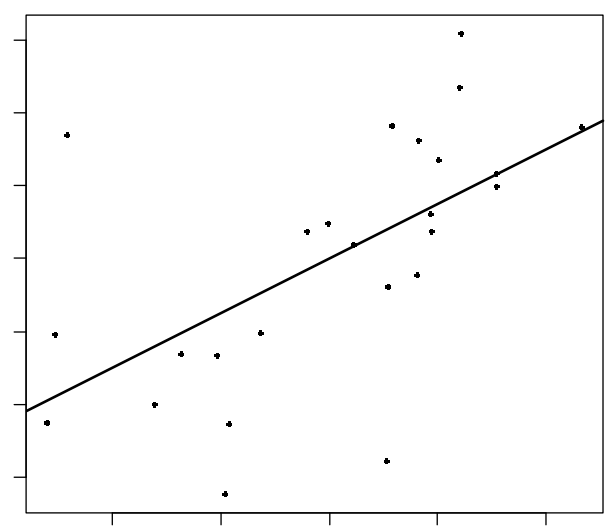

ers

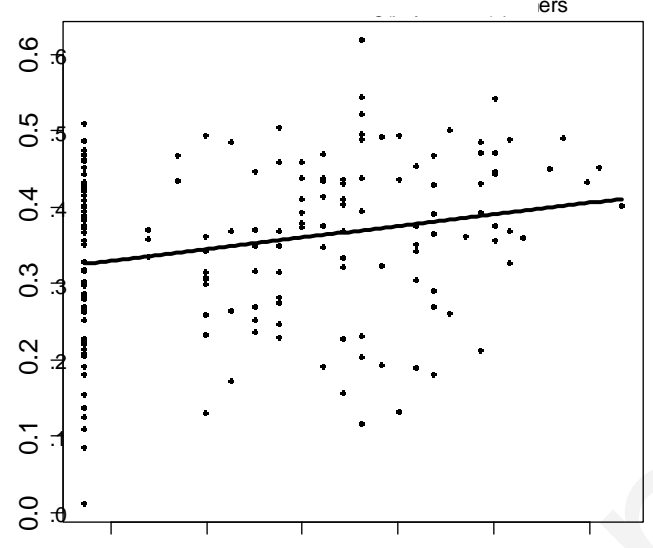

'en

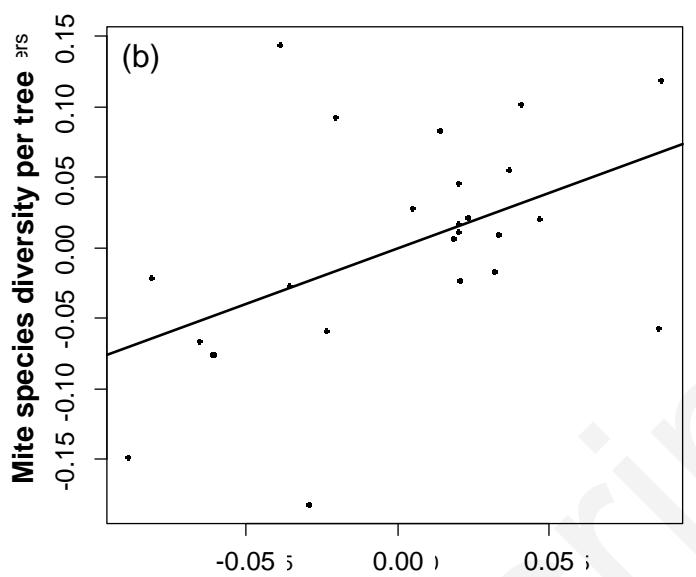

Habitat diversity per tree thers

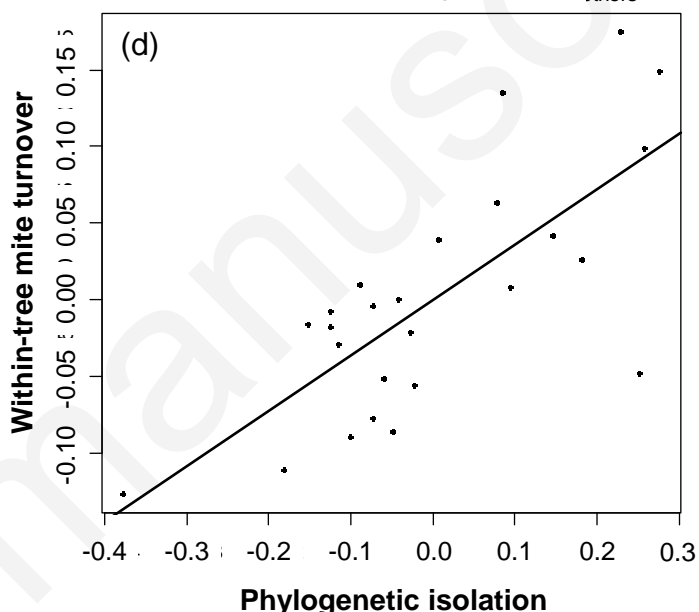

Figure 2. 


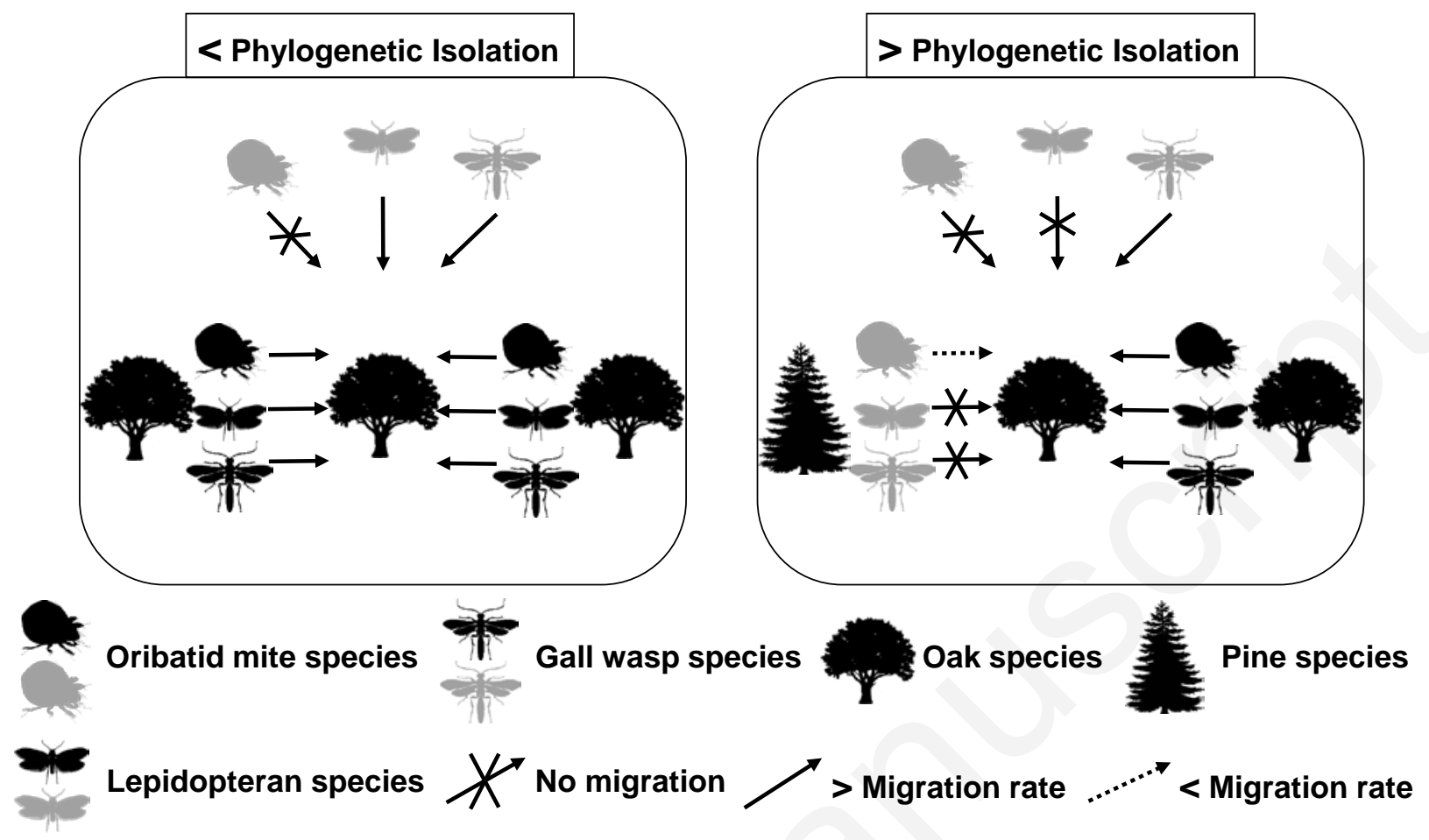

Figure 3. 


\section{FIGURE LEGENDS}

Figure 1. Sampling scheme established in a temperate mixed forest located close to Rennes, Bretagne, France (48.11 N, -1.34 W): (a) Triplet of oaks (dark trees) varying in age (trunk width) and phylogenetic distance of neighbours (trees of other colours and shapes). (b) Three strata within an oak crown (upper, lower shaded, lower more exposed). (c) younger and older branches within strata. Oak trees belong to Quercus petreae and $Q$. robur species. Neighbour trees also belonged to the following species: Ilex aquifolium, Fagus sylvatica, Castanea sativa, Ulmus minor, Alnus glutinosa, Sorbus torminalis, Corylus avellana, Carpinus betulus, Populus tremula, Salix caprea, Abies alba, Rhamnus frangula, Tilia cordata, Betula pendula, Prunus avium, Malus sylvestris and Pyrus pyraster.

Figure 2. Partial residual plots showing the effects of (a) tree phylogenetic isolation and (b) habitat diversity per tree on mite alpha-diversity per tree, (c) of branch age on mite species diversity per branch, and (d) of phylogenetic isolation on within-tree mite turnover. Partial residual presents the response of a given dependent variable to a given predictor variable while accounting for the effects of other predictor variables in the multiple regression models (see Table 2). Variables were In+1-transformed.

Figure 3. Observed effect of host phylogenetic isolation on the assembly of different groups of colonizers. A phylogenetically isolated host (right) may present higher species diversity of oribatid mites due to immigration, even at low rate, of new species (grey) from distantly related host neighbours, while there is no long-distance immigration from distant closely related host trees (long-distance immigration is represented on top of figure). On the other hand, host phylogenetic isolation may have no effects on gall wasps due to their high dispersal and habitat manipulation capacities, which allow them to colonize across large distances any tree individual to which they are specialized. Finally, the diversity of lepidopterans may be negatively affected by phylogenetic isolation of a focal tree because lepidopterans on neighbouring trees cannot use the 
focal tree nor can they manipulate its nutritional quality (as suggested by Yguel et al., $2011,2014)$. Lack of colonization from distantly related neighbours is not compensated by increased immigration across large distances from closely related host trees.

Silhouettes of oribatids (by B. Lang) and gall wasps (by M. Broussard), and lepidopterans (uncredited image) and pines (uncredited image) from http://phylopic.org, respectively under licenses of http://creativecommons.org/licenses/by/3.0/, and http://creativecommons.org/publicdomain/mark/1.0/. 